\title{
Kant, Schlick and Friedman on Space, Time and Gravity in Light of Three Lessons from Particle Physics
}

\author{
J. Brian Pitts ${ }^{1}$ D
}

Received: 28 March 2016/Accepted: 21 January 2017/Published online: 2 March 2017

(C) The Author(s) 2017. This article is published with open access at Springerlink.com

\begin{abstract}
Kantian philosophy of space, time and gravity is significantly affected in three ways by particle physics. First, particle physics deflects Schlick's General Relativity-based critique of synthetic a priori knowledge. Schlick argued that since geometry was not synthetic a priori, nothing was-a key step toward logical empiricism. Particle physics suggests a Kant-friendlier theory of space-time and gravity presumably approximating General Relativity arbitrarily well, massive spin2 gravity, while retaining a flat space-time geometry that is indirectly observable at large distances. The theory's roots include Seeliger and Neumann in the 1890s and Einstein in 1917 as well as 1920s-1930s physics. Such theories have seen renewed scientific attention since 2000 and especially since 2010 due to breakthroughs addressing early 1970s technical difficulties. Second, particle physics casts additional doubt on Friedman's constitutive a priori role for the principle of equivalence. Massive spin-2 gravity presumably should have nearly the same empirical content as General Relativity while differing radically on foundational issues. Empirical content even in General Relativity resides in partial differential equations, not in an additional principle identifying gravity and inertia. Third, Kant's apparent claim that Newton's results could be known a priori is undermined by an alternate gravitational equation. The modified theory has a smaller (Galilean) symmetry group than does Newton's. What Kant wanted from Newton's gravity is impossible due its large symmetry group, but is closer to achievable given the alternative theory.
\end{abstract}

J. Brian Pitts

jbp25@cam.ac.uk

1 Faculty of Philosophy, Sidgwick Avenue, University of Cambridge, Cambridge CB3 9DA, UK 


\section{Introduction}

Reliable scientific knowledge should not depend strongly on accidents, or at least on not accidents that lead us to misjudge how evidence supports our theories. Failure of imagination can lead to our not entertaining theories that are comparably good to the ones that we did entertain; such unconceived alternatives undermine scientific realism (van Fraassen 1989, p. 143) (Sklar 1985; Stanford 2006; Roush 2005; Wray 2008; Khalifa 2010; Pitts 2016e). This problem is rendered systematic by the fact that, as shown in Bayesianism, scientific theory testing is comparative (Shimony 1970; Earman 1992; Sober 2008; Pitts 2013). How well the evidence $E$ fits my favorite theory $T$ depends, perhaps surprisingly, on how likely some other theory $T_{1}$ (indeed all other theories, including ones that I haven't thought of) makes the evidence $E$ and how I spread my degrees of belief among the other theories $T_{1}, T_{2}$, etc. One sees this fact by expanding the denominator $P(E)$ of Bayes's theorem using the theorem of total probability:

$$
P(E)=P(E \mid T) P(T)+P\left(E \mid T_{1}\right) P\left(T_{1}\right)+P\left(E \mid T_{2}\right) P\left(T_{2}\right)+\ldots
$$

In the interest of freeing ourselves from historical accidents regarding space-time theory, it is prudent to employ whatever systematic means exist for generating plausible alternative theories.

Fortunately, there is a largely untapped source here, the literature that studies all possible classical (non-quantum) relativistic wave equations; it has gone untapped for a number of reasons, including a superficially quantum vocabulary. That literature is particle physics, of which the late 1930s taxonomy of relativistic wave equations in terms of mass and spin [e.g., (Pauli and Fierz 1939; Fierz and Pauli 1939; Wigner 1939)] is a prominent example. In 1939 particle physicists Wolfgang Pauli and Markus Fierz began to subsume Einstein's prematurely invented (Ohanian 2008, p. 334) General Relativity within the particle physics taxonomy as massless and spin-2 (Pauli and Fierz 1939; Fierz and Pauli 1939). Pauli and Fierz's work also makes it natural to consider a small non-zero mass and spin-2 as a potential alternative theory, one which [as Seeliger understood in a simpler example already in the 1890s (von Seeliger 1895; Norton 1999)] presumably would approximate General Relativity as closely as desired. This expectation was so overwhelmingly natural that its failure (at least with approximate calculations) discovered in 1970 was a "bombshell" (Deser 1971).

\subsection{Particle Physics Background}

Pondering Maxwell's electromagnetism and Einstein's General Relativity, general relativists and philosophers often discuss relativistic wave equations in which the waves travel at the 'speed of light.' In particle physics it is routine to consider also wave equations for some particle/field(s), such as electrons, (some?) neutrinos, the weak nuclear force $W^{ \pm}$and $Z$ bosons, and maybe even light and/or gravity themselves, that include an algebraic term in a field potential $\phi$ in the field equations. The coefficient of such an algebraic term is the "mass" (squared) of the 
particle/field $\phi$. Such terminology makes inessential use of Planck's constant; I set the reduced version $\hbar$ to 1 . The "mass" is in effect an inverse length scale, which one could take to be primitive, avoiding the appearance of Planck's constant (and the speed of light in that term). The resulting wave equation, invented multiple times around 1926 (Kragh 1984), is generally known as the Klein-Gordon equation

$$
\left(-\frac{1}{c^{2}} \partial^{2} / \partial t^{2}+\nabla^{2}-m^{2} c^{2}\right) \phi=0 .
$$

(Having displayed the speed of light $c$, I now set it to 1 as well.) "Particle mass" in that sense is just a property of a classical field, an inverse length scale, expressed in entrenched quantum terminology for which there is no convenient alternative. In the static, spherically symmetric case, this equation becomes

$$
\left(\nabla^{2}-m^{2}\right) \phi=\frac{1}{r^{2}} \frac{\partial}{\partial r}\left(r^{2} \frac{\partial \phi}{\partial r}\right)-m^{2} \phi=0 .
$$

For a massive theory, one gets a faster, exponential fall-off as $\frac{1}{r} e^{-m r}$. For wave solutions satisfying the Klein-Gordon equation, energy propagates (at the group velocity) more slowly than light at a speed(s) depending on $m$ and the frequency(s).

A potential of the form $\frac{1}{r} e^{-m r}$ appeared in the 1890s in astronomy and physics independently in the work of Neumann and Seeliger (Neumann 1886, 1896, 1903; Pockels 1891; von Seeliger 1896; Norton 1999; Pitts 2016d) and again due to Yukawa in particle physics in the 1930s (Yukawa 1935). The inverse of $m$ is known as the range of the field, so nonzero $m$ gives a field a finite-range, while $m=0$ gives a "long" or "infinite" range. Seeliger and Neumann provided an alternative to Newton's theory by 1900 , Seeliger providing cosmological motivations to make the gravitational potential converge in an infinite homogeneous universe and Neumann providing an appropriate partial differential equation and its solution. Neither had much to say about the physical meaning of the new parameter. That lack of physical meaning and connection to other experience was noticed and faulted by Schlick (Schlick 1920, p. 70). That lacuna was filled in the 1920s, however, making that aspect of Schlick's critique obsolete quickly, at a time when the contest between broadly Kantian and positivist conceptions of philosophy was still live. That altered situation in physics unfortunately went unrecognized in philosophy, however.

In the late 1930s Pauli and Fierz found that the theory of a non-interacting massless spin 2 (symmetric tensor) field in Minkowski space-time was just the linear approximation of Einstein's General Relativity (Pauli and Fierz 1939; Fierz and Pauli 1939). Inspired by de Broglie and Pauli-Fierz, Marie-Antoinette Tonnelat and Gérard Petiau explored massive graviton theories on a sustained basis during the 1940s (cited below). Massive theories are plausible in terms of relativistic field theory. Such work reached maturity (partly in other hands) in the 1960s (Ogievetsky and Polubarinov 1965; Freund et al. 1969). As Freund, Maheshwari and Schonberg put it,

In the Newtonian limit, Eq. (1) is now replaced by the Neumann-Yukawa equation, 


$$
\left(\Delta-m^{2}\right) V=\kappa \rho,
$$

which leads to the quantum-mechanically reasonable Yukawa potential

$$
V(r)=-\frac{\kappa M e^{-m r}}{r},
$$

...(Freund et al. 1969).

This potential was sufficiently plausible as to be independently invented 3 times [Seeliger among many other potentials, Neumann, and Einstein in 1917 on the way to inventing his cosmological constant $\Lambda$ (Einstein 1923)]. Seeliger and Einstein were both addressing the problem of mathematically divergent gravitational potential in an infinite homogeneous static Newtonian universe. Unfortunately philosophy never paid attention to massive spin-2 gravity, and hence failed to realize that Einstein's theory had serious competition (in the sense of decent prior probability, rendering the data nearly as likely as General Relativity did for all that anyone knew, and making a radical conceptual difference to space-time philosophy-a sort of philosophical expected utility) up to 1970 at least. Massive photon theories are fine even when merged with quantum mechanics to obtain massive quantum electrodynamics (Belinfante 1949) [for many references see (Pitts 2011b)].

However, in the early 1970s, massive gravitons, which one would expect to behave analogously, ran into serious trouble on detailed technical grounds (van Dam and Veltman 1970, 1972; Boulware and Deser 1972). It was concluded that every such theory suffered from either an empirical problem due to a discontinuous massless limit $m \rightarrow 0$ (for pure spin 2) or a problem of violent instability (for spin 2 and spin 0 together, because the spin 0 (scalar) degrees of freedom have negative energy, so in quantum field theory one would expect explosive spontaneous creation of positive-energy spin 2 and negative-energy spin 0 gravitons). Moreover, a theory that appeared to suffer from the former problem in the lowest approximation turned out to have the latter problem (in addition or instead) when treated exactly (Tyutin and Fradkin 1972; Boulware and Deser 1972), a problem recently dubbed the "Boulware-Deser ghost." Philosophers and historians who take the General Relativity side of the General Relativity versus particle physics rift in physics (Feynman et al. 1995; Rovelli 2002) -which is most of them, often perhaps unwittingly-had gotten lucky. The serious rival theories that they never contemplated, turned out not to work after all, the 1970s showed (or so it seemed). Sometimes what you don't know won't hurt you.

It's not a reliable principle of scientific method, however, and in this instance much of the original evidence has collapsed. The tide has turned and massive graviton theories have been widely studied lately by physicists, who now know much about how to solve both the empirical discontinuity problem (partly with the help of numerical simulation) (Vainshtein 1972; Deffayet et al. 2002) and how to solve the instability problem (de Rham et al. 2011; Hassan and Rosen 2011, 2012; Hinterbichler 2012). The competition between General Relativity (self-interacting universally coupled massless spin-2) versus massive gravity (self-interacting universally coupled massive spin-2) is a well motivated example of the fact, noted 
by Pierre Duhem, that the curve fitting problem always applies in physics: through any set of experimental results, multiple curves can be proposed (Duhem 1954).

\subsection{Outline}

This paper will discuss three interrelated themes involving the surprising relevance of particle physics, in particular massive theories of gravity, to the well-studied Kant-Einstein interface. First, Schlick's critique of Kant in light of General Relativity will be seen to be less than compelling once one clearly entertains the possibilities recognized in particle physics. These possibilities partly predated Schlick's critique of Kant, but were fully developed later because Einstein developed his field equations earlier than the natural development of physics should have produced. Second, Friedman's invocation of a constitutive a priori role for the principle of equivalence, which has been criticized already by Howard (2010), will be seen to be less than compelling in light of particle physics. Particle physics shows how to construct a theory that, one would expect, distinguishes inertia from gravitation, while empirically approximating Einstein's equations as closely as one wishes. Third, though the symmetry group of Newtonian physics is much larger than Kant recognized, particle physics provides an alternate theory that reduces that gap.

\section{Massive Gravity Versus Schlick's Critique of Kant from General Relativity}

Moritz Schlick, future leader of the Vienna Circle, argued around 1920 that General Relativity made even a broadly Kantian philosophy of geometry impossible because the physical truth about the actual world was incompatible with it (Schlick 1920, 1921; Coffa 1991; Friedman 2002; Ryckman 2005). If even geometry is not an example of synthetic a priori knowledge, then nothing is. Ryckman has usefully framed the widely accepted view of the destructive significance of General Relativity for Kantian philosophy:

Kantian and neo-Kantian publications comprised a not-insignificant torrent in the "relativity rumpus" following the announced confirmation of the general theory of relativity in November 1919. [footnote suppressed] ...[I]t was incontrovertible that general relativity, on corroboration of the dramatic prediction of star images displaced by the sun's gravitational field, minimally required modification or clarification of the necessarily Euclidean structure of space implied by the Transcendental Aesthetic. Most of this literature, regardless of its provenance, contains little of present interest. But within a few months in late 1920 and early 1921, Ernst Cassirer and Hans Reichenbach published neo-Kantian appraisals of the theory of relativity whose historical and philosophical significance has acquired renewed relevance at the beginning of the 21st century. [footnote suppressed] (Ryckman 2005, pp. 13, 14) 
Some of these nameless Kantians and neo-Kantians are discussed by Howard (1994, 2010). Cassirer's and Reichenbach's appraisals involve massive retrenchment. Was that really necessary due to scientific progress?

There is a sense in which philosophers should not be expected solve that problem. It just isn't part of their professional training or responsibility to propose scientific theories, so if scientists don't propose the theories that philosophers need, who will? One might hope that someone knowledgeable about both subjects (perhaps someone like Schlick?) would take up that task. But Schlick, as will appear, was too partisan to fill that role. Someone who paid attention to what one could start to identify as particle physics in the 1920s [perhaps de Broglie or one of the many inventors of the Klein-Gordon equation (Kragh 1984)] could have given Kantian philosophers a friendly tip. But that didn't happen, either, it seems (Hentschel 1990). Thus the available scientific resources for maintaining a Kantian position simply went unrecognized for a very, very long time, long past the time that many people cared about a Kantian position, in fact.

The question of the degree to which the progress of science is inevitable or contingent has received some attention (Hacking 2000; Soler 2008). What has not been noticed is that Ryckman's widely shared assessment of the impact of General Relativity on the family of Kantian philosophies, in retrospect, was an historical accident. Lakatos's point that the actual contingent history must be held to normative standards in order to discern scientific progress (Lakatos 1970, 1971) is borne out especially in the context of the problem of unconceived alternatives or underconsideration [e.g., (Stanford 2006)]. Indeed even a real historian of science, Kuhn (as opposed to Lakatos), agreed with Lakatos that historians should be prepared to identify historical actors' mistakes and that doing so was often important and illuminating (Kuhn 1971).

\subsection{Did Light Bending Verify General Relativity?}

It is striking how routinely the 1919 observed bending of light is construed as a verification of General Relativity. Impressive confirmation, yes, but verification? That is a methodological holdover from nineteenth century Baconianism, contrary to Duhemian underdetermination, Popperian falsificationism, the promising parts of logical empiricist confirmation theory, Bayesianism, etc. While one can forgive the enthusiasm of the popular media in 1919 in the aftermath of the Great War (Pais 1982), the New York Times should not be allowed to distort space-time philosophy permanently. Yet philosophers of science have been slow to apply standard philosophy of science ideas to the bending of light. It is fair to say that the bending of light falsified Nordström's scalar theory of gravity (Kraichnan 1955; Pitts 2016d). Using 1920s-30s mathematics, one can make the failure to bend light in Nordström's theory (Einstein and Fokker 1914; von Laue 1917) manifest in that the part of the effective space-time geometry that light sees is untouched by gravity according to Nordström's theory (Pitts 2016d). (It is difficult to imagine a plausible Duhem-Quine rescue story in this case.)

But surely other theories, perhaps not yet proposed and possibly not so revolutionary, might also predict the bending of light, so that it could not verify 
General Relativity? So, evidently, many physicists reasoned in the 1920s (Brush 1989). It appears that for many people, including Bertrand Russell, Whitehead's theory (Whitehead 1922; Eddington 1924) filled that role (Russell 1927). Massive gravity should have filled that role, especially from 1939 onwards. Whitehead's is not even a field theory, but rather a theory with retarded action at a distance in flat Minkowski space-time. Despite the theoretical backwardness of action at a distance, Whitehead's theory was empirically viable still in the 1950s (Schild 1956). It is wholly appropriate that a role for a more conservative theory of gravity and spacetime than Einstein's was recognized, but filling it with only Whitehead's theory made the philosophical theses made to hang upon that role needlessly fragile.

Another source of confusion is Einstein's mistaken analogy between his cosmological constant $\Lambda$ and his 1917 reinvention of the Seeliger-Neumann finite-range modification of Newtonian gravity (Heckmann 1942; Trautman 1965; Schucking 1991; Harvey and Schucking 2000; Norton 1999; Earman 2001). This faulty analogy has deceived many serious writers for a long time, including North (1965, p. 179, 1994, pp. 515, 516), Jammer (1993, pp. 194, 195), Pais (1982, p. 286), and Kragh (2004, p. 28). The mistake happens in the middle of Einstein's cosmological constant paper, between the scalar and tensor sections. That false analogy almost certainly helped to delay the conception of massive spin-2 gravity by decades. How could one think to do for the first time what Einstein supposedly had already done? Or does Einstein's theory win forever because it was the first tensor theory of gravity, the kind of theory that can bend light?

\subsection{Schlick's Contribution}

Howard has outlined several useful themes:

Schlick was one of the first philosophers to consider carefully the philosophical implications of relativity, and in Einstein's opinion, his analysis was far superior to those of most other philosophers because he did not try to appropriate the relativity theory to a partisan philosophical cause (as many neo-Kantians and positivists had done). Schlick also brought to his work a better grounding in physics than most other philosophers of his day could claim, for in 1904 he had taken a degree in physics under Max Planck at Berlin. Some years later, Schlick became the logical positivist we know as the founder of the Vienna Circle. But in 1915 Schlick's philosophy of science was a novel combination of realistic and conventionalistic components.

Schlick's first essay on relativity (1915) was published late in the same year in which Einstein completed his work on general relativity. The main purpose of the essay was to criticize the neo-Kantian and positivistic misinterpretations of relativity, and to exhibit, by way of contrast, some of the main philosophical implications that would be revealed by an unprejudiced reading of the theory. (Howard 1984)

I suggest that Schlick felt less temptation to force-fit an alien philosophy onto General Relativity than did other philosophers because his philosophy already was 
more or less congenial to General Relativity. Einstein and Schlick had been drinking from many of the same wells, including Mach. Perhaps Schlick's superior interpretation of General Relativity owes somewhat less to fair-mindedness than might otherwise be inferred, however. To ascertain whether Schlick was less partisan as a philosophical commentator on space-time physics, one should look more broadly: how well did he use his superior knowledge of physics to illuminate the philosophical discussion? More subtly, did he help other philosophers scientifically in a way that he was unusually, perhaps uniquely qualified to do? Did he notice philosophically interesting holes in the scientific literature and fill them-even if not congenial to his own philosophical projects? Or did he use his expertise to claim beyond desert that science supported his philosophy? Lawyers are unusually persuasive at making arguments, but in an adversarial system the prosecutor or defense attorney (unlike a judge) only takes one side.

As it turns out, Schlick's making plausible that General Relativity refutes Kant was an accident. It depended crucially upon Schlick's philosophically partisan failure to apply his training as a physicist to ascertain whether the views that he wanted to undermine could be defended from his criticisms. Had he (or someone else) thought to propose it, it would have been easy to do to Einstein's theory what Seeliger, Neumann, and recently Einstein himself in 1917 (Einstein 1923) had done to Newton's theory, thereby producing a Kant-friendlier theory that was presumably practically empirically equivalent to General Relativity. Somewhat similar conceptual ingredients were available from Lotze (Lotze 1879), who seems to have been curiously ignored in the relativity debate (Hentschel 1990) despite having said Poincaré-like things in defense of Kant on geometry well before Poincaré (Torretti 1978b, 288, 289, 408; Lotze 1879, pp. 248, 249; Poincaré 1902). Lotze, however, had no scientific theory and rendered the true geometry of space undetectable, as opposed to merely locally undetectable but indirectly globally discernible. Analogous moves to Seeliger and Neumann soon would be made in electromagnetism by de Broglie, Proca and others in the 1920s-1930s. Such a proposal would not, of course, have suited Schlick's revolutionary project. His antiKantian message and pro-Machian sympathies would be have been muted by even a hint of the possibility of a modification of Einstein's equations which would approximate General Relativity as closely as desired, but containing a flat background metric that was observable in principle on astronomical scales and hence clearly real (albeit largely obscured by the distorting effects of gravity). Galileo often ignored Tycho; would Galileo have invented Tycho's theory out of fairness if Tycho hadn't already done so? Why look for alternatives that reduce the anti-Kantian sting of General Relativity to that of mere Special Relativity, when one has a partisan stance to defend? There were progressive cultural implications in a Weimar climate of controversy and incipient reaction (Okruhlik 2004).

If neo-Kantian philosophy of geometry was overthrown (historically-sociologically at least) by General Relativity due to arguments akin to Schlick's, then this is a good example of the problem of unconceived alternatives. The main problem for Kantian philosophy was a lack of timely love from good physicists who could have proposed a scientifically serious and Kant-friendlier theory of space-time and gravity. In the heyday of the debate there was not much chance that philosophers 
could identify the potential philosophical utility of incipient particle physics when even physicists had not done much in that direction. Much later but still long ago by now, things were different (Freund et al. 1969).

The untimely death of Poincaré (1912, age 58) is worth recalling here; it is easy to imagine Poincaré making such proposals in partial vindication of his conventionalist philosophy of geometry (Poincaré 1913; Walter 2010). It appears that much of 20th century philosophy was degraded by Poincaré's death. While people continued to talk about him, apparently no one followed him in a way that was both faithful and intelligent. Eddington, who had written a glowing obituary of Poincaré (Eddington 1913), later changed allegiance to Einstein and singled out only the most vulnerable parts of Poincaré's view as representative (Eddington 1920). Logical empiricists could call themselves conventionalists but differed from Poincaré on a number of points, not always for the better. Dingler's scientific intransigence (Torretti 1978a) made his profession of conventionalism more a liability than an asset.

It is widely believed that Schlick's work deploying General Relativity against neo-Kantian philosophy of geometry was both first-rate scientifically and philosophically at the time and of lasting significance. Alberto Coffa thought so:

Schlick was probably the first major philosopher to draw the philosophical lessons of relativity.... Now the theory of [general] relativity had forced his attention to the question of whether there is an apodictic a priori. A careful, prolonged analysis of the situation finally led him to conclude that there is no such thing and, more importantly, that this would entail a decisive break with the Kantian tradition. Schlick was the first one of the scientifically oriented neo-Kantians to understand that the philosophical lessons of relativity demanded not the correction but the elimination of Kantianism. (Coffa 1991, pp. 196, 197) (emphasis added to highlight success terms)

This is the language of a monument to a lasting achievement.

I suggest a different picture of this part of Schlick's work: scientifically serviceable in its own time, but partisan in using his scientific credibility to advance a philosophical agenda without making a scientific critique that he but not his opponents were capable of making - and also obsolete during the 1920s (not that this was pointed out). Thus he created a facade that his philosophical opinions were entailed by scientific results, when instead he could have proposed a partly new theory using off-the-shelf ingredients that would have leveled the playing field. His breathless endorsement of Einstein at the start of his own book (Schlick 1920) and hymn to the universe, General Relativity and Einstein (below) (Schlick 1920, pp. $74,75)$ do not suggest that the author was much interested in the epistemic caution involved in cultivating alternate theories. He was far from Popper's critical spirit. Furthermore, his work was obsolete when massive spin 2 gravity and the recognition of General Relativity as a massless spin 2 theory (Pauli and Fierz 1939; Fierz and Pauli 1939) made it obvious how to start writing down a theory that, one would expect, would fit the data as well as Einstein's while having a fixed a priori background geometry that is, in principle, observable astronomically. There were clues already in 1917 and more in the 1920s. 


\subsection{Lindemann's Challenge, Answered}

One gets clear answers to the issues raised in the introduction by F. A. Lindemann for the English translation of Schlick, issues for philosophers, presumably especially Kantians, who wanted to preserve more traditional views about space and time. Here is Lindemann's challenge.

The main achievement of the general theory of relativity has caused almost more difficulty to the school of philosophers, who would like to save absolute space and time, than the welding of space and time itself. Briefly this may be stated as the recognition of the fact that it is impossible to distinguish between a universal force and a curvature of the space-time-manifold, and that it is more logical to say the space-time-manifold is non-Euclidean than to assert that it is Euclidean, but that all our measurements will prove that it is not, on account of some hypothetical force....

At first sight it might appear that there must be an easy way to settle the question. The golfer [who finds that balls spiral into the hole, despite his inclination to believe the green level] has only to fix three points on his putting-green, join them by straight lines, and measure the sum of the three angles between these lines. If the sum is two right angles the green is flat, if not, it is curved. The difficulty, of course, is to define a straight line. If we accept the definition of the shortest line, we have carried out the experiment, for the path of a ray of light is the shortest line and the experiment which determines its deflection may be read as showing that the three angles of the triangle - star-comparison star-telescope - are not equal to two right angles when the line star-telescope passes near the sun. But some philosophers appear not to accept the shortest line as the straight line. What definition they put in its place is not clear, and until they make it clear their position evidently is a weak one. It is to be hoped they will endeavour to do this, and to explain the observed phenomena rather than adopt a merely negative attitude. (Schlick 1920, pp. iv-vi)

That was a well-framed and intellectually reasonable view in 1920, though not compelling for all rational beings. Whether it was professionally reasonable to demand that philosophers make a novel contribution to physics and mathematics is harder to say, unless they were brought up as physicists first like Schlick. LeviCivita's general bimetric geometry, for which such nameless philosophers seem to have been groping, still lay in the future (Levi-Civita 1926) (though many special cases involving flat and conformally flat geometries already were known (Pitts 2016d), and they suffice to make the conceptual point, though not to express an adequate theory of space-time and geometry in 1920). But by now Lindemann's requests have been fulfilled, if not by philosophers, then by the particle physics tradition, and some of the key materials for doing so existed well before Lindemann wrote. The massless spin 2 derivations of Einstein's equations from flat space-time would eventually (Kraichnan 1955; Gupta 1954; Feynman et al. 1995; Weinberg 1964a, b; Deser 1970; Pitts and Schieve 2001) show why it might not be 
unreasonable to favor universal forces even given Einstein's equations because it isn't implausible that gravity would act in just that way without any peculiar premises. [Such derivations turn out to be built around what one can recognize as the converse of Noether's Hilbertian assertion (Pitts 2016a).] Better yet, the already extant Neumann-Seeliger-Einstein 1890s/1917 modification of Newtonian gravity in principle showed the way to taking massive spin-2 gravity (not a hypothetical force) to be an almost-universal force (Ogievetsky and Polubarinov 1965; Freund et al. 1969), in the sense of acting like a Poincaré-Reichenbach universal force if one is unable to perform experiments sensitive to long-range gravitational effects. Like their contemporary the Ford Model T, Lindemann's views, if construed as unanswerable rhetorical questions, are now somewhat dated.

\subsection{Neglect of Lotze}

\section{Recall that General Relativity is viewed as making it}

incontrovertible that general relativity, on corroboration of the dramatic prediction of star images displaced by the sun's gravitational field, minimally required modification or clarification of the necessarily Euclidean structure of space implied by the Transcendental Aesthetic. (Ryckman 2005, pp. 13, 14)

If such scientific assistance as I have envisaged had been available, as it easily could have been, then not much clarification of Kant's philosophy would have been required beyond that already achieved in embryo by Lotze in his brief best moments (Lotze 1879 , pp. 248, 249), on top of whatever adjustments were required in updating Kant from space to space-time to fit special relativity. According to Torretti,

Lotze was, as far as I know, the first one to make the following important remark, which Poincaré later used in support of conventionalism. In Euclidean geometry, the three internal angles of a triangle are equal to two right angles. This fact, Lotze claims, is not subject to experimental verification or refutation. If astronomical measurements of very large distances showed that the three angles of a triangle add up to less than two right angles, we would conclude that a hitherto unknown kind of refraction has deviated the light-rays that form the sides of the observed triangle. In other words, we would conclude that physical reality in space behaves in a peculiar way, but not that space itself shows properties which contradict all our intuitions and are not backed by an exceptional intuition of its own. ${ }^{1}$ (Torretti 1978b, 288, 289)

The additional ingredients needed beyond Lotze were supplied by Neumann, Seeliger and Einstein's modification of Newtonian gravity. Doing to General

\footnotetext{
${ }^{1}$ käme es aber einmal dazu, daß astronomische Messungen großer Entfernungen nach Ausschluß aller Beobachtungsfehler eine kleinere Winkelsumme des Dreiecks nachwiesen, was dann? Dann würden wir nur glauben, eine neue sehr sonderbare Art der Refraction entdeckt zu haben, welche die zur Bestimmung der Richtungen dienenden Lichtstrahlen abgelenkt habe; d.h. wir würden auf ein besonderes Verhalten des physischen Realen im Raume, aber gewiß nicht auf ein Verhalten des Raumes selbst schließen, das allen unseren Anschauungen widerspräche und durch keine eigene exceptionelle Anschauung verbürgt würde. (Lotze 1879, pp. 248, 249; Torretti 1978b, p. 408).
} 
Relativity what those three had done to Newton's theory would restore a flat background space-time geometry, one difficult to observe due to gravity's almostuniversal distortion effects, but observable on long distances due to the new term (eventually interpretable as a graviton mass) in the field equations.

\subsection{Neglect of Massive Gravity}

Was massive gravity or something like it part of the discussion when philosophers were pondering General Relativity? It was not. Probably the closest that one can find is a near-miss in the work of Peter Mittelstaedt (Mittelstaedt 1970, 1976). It is notorious in physics that general relativists and particle physicists do not tend to interact profitably regarding gravitational physics (Feynman et al. 1995; Preskill and Thorne foreword) (Rovelli 2002; Brink 2006). It is equally clear, if one knows what to look for, that the literature in the history and philosophy of space-time and gravity tends to ignore the particle physics side. Conveniently enough, one can identify a couple of sources that are sufficiently comprehensive that, if massive gravity had been part of the discussion, then those sources most likely would have noticed it. Hentschel's massive study helpfully includes a section on other theories entertained at the time. One of the key features, at least to the particle physicstrained eye, is a privation: nothing like massive gravity appears (Hentschel 1990, pp. 46-54). Neither does the name of Markus Fierz, Pauli's collaborator in identifying the linearized source-free Einstein's equations as massless spin 2, appear in the index or in the bibliography. Another quite comprehensive source likely to mention massive gravity if it had arisen is Combridge's bibliography (Combridge 1965), but evidently it did not arise in the literature of 1921-1937.

If anyone had paid attention both to particle physics and to the status of Kantian philosophy vis-a-vis General Relativity, an obvious question would have been, "why not do to Einstein's theory what Seeliger, Neumann and Einstein did to Newton's?" Such a question should have been all the easier to ask once the exponentially decaying potential had a physical meaning as a graviton mass in the 1920s-30s, as opposed to the bare parameter in Neumann's, Seeliger's and Einstein's works. One can see from Hentschel's work that no such thing happened (Hentschel 1987, 1990). Evidently even ideas in the neighborhood of Lotze's were not entertained much. While the bibliography contain's Lotze's Grundzüge der Naturphilosophie, his name does even not appear in the exhaustive index, much less the relevant sections. If we can excuse the historical actors of the 1920s-1930s, it isn't necessary to follow them.

In one sense something like massive gravity was already part of the discussion, namely, Schlick's discussion of Seeliger (Schlick 1920, ch. 9, p. 70)-but this section soon become scientifically obsolete. Recognizing the problem of the divergent gravitational potential for an infinite homogeneous Newtonian universe and Seeliger's solution to it, Schlick says only this: “An unsatisfactory feature of this theory is, however, contained in the fact that the hypothesis is invented ad hoc, and is not occasioned or supported by any other experience." Schlick, it is noteworthy, makes his own judgment of Seeliger rather than being misled by Einstein's false analogy. Schlick's judgment is far from prophetic in insight, 
however. While his complaint is true of many of Seeliger's ad hoc force-laws, it is less true of the Neumann-Einstein modification of the Poisson equation. More to the point, once the idea that matter is fields and hence must satisfy relativistic wave equations caught on - an idea with antecedents in the 1910s electromagnetic world picture and more clearly evident in Pascual Jordan in the late 1920s- the ubiquity of slow-moving matter (rocks, trees, tables, buildings, etc.) implied that there was an enormous amount of experience supporting rest mass terms for matter fields at least by the late 1930s. It is plausible by analogy that electricity or gravity might have the same feature. Not coincidentally, such a development is one of the noteworthy features of 1920s-1930s physics (de Broglie's massive photons, the Klein-Gordon equation, the Yukawa potential, Proca's massive vector meson field, Wigner's mass-spin taxonomy, etc.). Thus there an overwhelming amount of experience of matter described by relativistic wave equations with mass terms, and the articulated theoretical possibility due to de Broglie from 1922 that electromagnetism had the same feature. Why shouldn't gravity also, a topic later considered in that light largely by Marie-Antoinette Tonnelat and Gerard Petiau on a sustained basis with de Broglie's involvement (Tonnelat 1941; Petiau 1941a, b; de Broglie 1943; Tonnelat 1943, 1944a, b, c; Petiau 1945; de Broglie 1954)?

Prior to his underestimate of Seeliger, Schlick had already made his own job easier by adopting a policy of dismissing the type of underdetermination-byapproximation worries that concerned Seeliger and Duhem. The merely approximate character of the confirmation of Special Relativity was no obstacle to accepting the theory as exact for philosophical interpretation (Schlick 1915, p. 159)! Such a claim seems to conflate interpreting a theory and interpreting the range of theories that fit the data at hand.

Such a claim might make sense if one is convinced that there exist no plausible and conceptually different theories that approximate the theory in question. The availability from the 1920s onward of the concept of massive theories, and especially recognition [probably from the 1930s (Fierz and Pauli 1939; Pauli and Fierz 1939)] of their tendency to have smaller symmetry groups than do massless theories, made it appropriate to recognize what Schlick hadn't envisaged, namely, plausible and conceptually distinct theories that approximate the theory in question. If the photon or graviton mass were just another really small parameter that might be 0 , it would be reasonable to ignore it. But since the photon or graviton mass is a physically meaningful concept, indeed one of a type that is exemplified for at least some other fields (the electron field is massive, for example, as are the weak force bosons), and since a photon or graviton mass breaks the gauge symmetry 'group' and hence makes a large conceptual difference, while a scalar graviton mass at least breaks the conformal group and leaves only the Poincare group, the conditions that license ignoring such rivals are not satisfied. Schlick (1920,1921), though trained as a physicist and hence capable of making his own assessments, failed to see the potential significance of Seeliger's work. Yet analogous ideas to the NeumannSeeliger mathematics would very shortly start to emerge independently in the work of de Broglie (de Broglie 1922, 1923, 1924) and others for massive particles/waves in the Klein-Gordon equation. But Schlick's unrevised views remained influential in philosophy. 


\subsection{Schlick's Hymn to the Universe, General Relativity, and Einstein}

By contrast, Schlick's enthusiasm for what Einstein achieved with the cosmological constant $\Lambda$ (Schlick 1920, pp. 70-75) is perhaps unparalleled by any subsequent writer, especially as shown in the hymn to the universe, General Relativity, and Einstein.

The structure of the universe, which the general theory of relativity unveils to us, is astounding in its logical consistency, imposing in its grandeur, and equally satisfying for the physicist as for the philosopher. All the difficulties which arose from Newton's theory are overcome; yet all the advantages which the modern picture of the world presents, and which elevate it above the view of the ancients, shine with a clearer lustre than before. The world is not confined by any boundaries, and is yet harmoniously complete in itself. It is saved from the danger of becoming desolate, for no energy or matter can wander off to infinity, because space is not infinite. The infinite space of the cosmos has certainly had to be rejected; but this does not signify such sacrifice as to reduce the sublimity of the picture of the world. For that which causes the idea of the infinite to inspire sublime feelings is beyond doubt the idea of the endlessness of space (actual infinity could not in any case be imagined); and this absence of any barrier, which excited Giordano Bruno to such ecstasy, is not infringed in any way.

By a combination of physical, mathematical, and philosophic thought genius has made it possible to answer, by means of exact methods, questions concerning the universe which seemed doomed for ever to remain the objects of vague speculation. Once again we recognize the power of the theory of relativity in emancipating human thought, which it endows with a freedom and a sense of power such as has been scarcely attained through any other feat of science. (Schlick 1920, pp. 74, 75)

Schlick seems not to have been disposed to use his training as a physicist to cultivate an unbiased range of scientific options for philosophical evaluation.

His remarks should be compared with another assessment of the cosmological constant $\Lambda$, namely, that it was difficult to interpret (Freund et al. 1969; McCrea 1971; Kerszberg 1989). The matter was well described by Freund, Maheshwari and Schonberg, who were not confused by Einstein's false analogy.

In the "Newtonian" limit it leads to the potential equation,

$$
\Delta V+\Lambda=\kappa \rho .
$$

Correspondingly, the gravitational potential of a material point of mass $M$ will be given by

$$
V=-\frac{1}{2} \Lambda r^{2}-\frac{\kappa M}{r}
$$


A "universal harmonic oscillator" is, so to speak, superposed on the Newton law. The origin of this extra "oscillator" term is, to say the least, very hard to understand. (Freund et al. 1969)

These remarks are not a bit poetic, but they are entirely reasonable by the standards of the late 1960s, a much better informed time than the late 1910s.

\subsection{Reichenbach and Carnap Did Not Alter the Situation}

One might think of Reichenbach or Carnap as taking Schlick's baton in paying ongoing philosophical attention to space, time and General Relativity. But on the issues at hand, Carnap and Reichenbach help to explain the persistence of the problem. Reichenbach, despite his impressive and sustained engagement with space-time theory and geometry throughout the 1920s, doesn't help much on this point. He has great praise for Schlick as philosophically unbiased and displaying a sure understanding of physics (Reichenbach 1978, pp. 36, 37). That is of course true if it means the absence of other philosophers' biases against General Relativity and having a comparatively solid understanding of that theory. Schlick's effort to tie Einstein's theory to Machian relationalism (Schlick 1920) seems to leave little room for gravitational radiation, however. Reichenbach makes no mention of Seeliger, Neumann, or Lotze. He remained intelligently engaged with space-time theory at least throughout the 1920s; his most serious work has appeared only in German and is hardly accessible (Reichenbach 1928, 1929a, b, appendix). ${ }^{2}$ He turned his attention largely elsewhere during the 1930s (Salmon 1977). Apparently he never noticed the relevance of particle physics and massive graviton theories. The fact that many of Reichenbach's minor works could be collected under the title Defending Einstein (Reichenbach et al. 2006) reminds us of the climate of contention and Reichenbach's role therein.

Carnap thought that Reichenbach had said what needed saying and hence quit writing on space-time (Carnap 1963, p. 957). Carnap presumably was complicit in the secret elimination of the lengthy appendix for the English translation (Reichenbach 1958), depriving readers of early 1920s developments in metricaffine geometry (showing the affine connection to be conceptually independent of the metric) and the question of chronogeometric significance. Yet Carnap's preface suggests that nothing important had happened in the 30 years since the (longer) German original appeared. With Carnap's imprimatur, four decades of physics (roughly 1918-1958) were written off for philosophers of space-time. The relevant science had terminated in Einstein's work, it now seemed.

\footnotetext{
${ }^{2}$ Recently an unpublished draft translation of the appendix of Philosophie der Raum-Zeit-Lehre, omitted from the published translation (Reichenbach 1958), appeared online (Reichenbach, nd). Thanks are due to Marco Giovanelli and to the Hans Reichenbach Papers at the Archives of Scientific Philosophy in the University of Pittsburgh library.
} 


\section{On Friedman's Constitutive a Priori Role of the Principle of Equivalence}

If massive gravity renders unclear the need for Schlick's General Relativity-based anti-Kantian revolution, it also sheds light on Michael Friedman's recent claim that the principle of equivalence plays a constitutive a priori role in General Relativitythat the principle of equivalence is required for the theory to have empirical content (Friedman 2001, 2002). (This critique of Friedman's treatment of the equivalence principle is complementary to Howard's critique (Howard 2010), for we reach similar conclusions by different but compatible arguments.) I have in mind "Einstein's principle of equivalence, which identifies gravitational effects with the inertial effects formerly associated with Newton's laws of motion..." (Friedman 2001, p. 37).

Such a variably curved space-time structure would have no empirical meaning or application, however, if we had not first singled out some empirically given phenomena as counterparts of its fundamental geometrical notions-here the notion of geodesic or straightest possible path. The principle of equivalence does precisely this, however, and without this principle the intricate spacetime geometry described by Einstein's field equations would not even be empirically false, but rather an empty mathematical formalism with no empirical application at all." (Friedman 2001, pp. 38, 39, footnote suppressed)

Later he reiterates the point:

in the absence of the principle of equivalence, Einstein's field equations remain a purely mathematical description of a class of abstract (semi-) Riemannian manifolds with no empirical meaning or application whatsoever. [footnote suppressed] (Friedman 2001, p. 81).

A bit later a weaker and more plausible claim is made:

Einstein's field equations are thus logically possible as soon as we have Riemannian manifolds available within pure mathematics, but they are only really possible (possible as an actual description of some empirical phenomena) when these abstract mathematical structures have been successfully coordinated with some or another empirical reality. [footnote suppressed] (Friedman 2001, p. 84).

While doubtless there is a job of coordination to do, and the principle of equivalence is a good way to do that job, the question is whether this principle of equivalence is unnecessarily strong. In fact without the principle of equivalence as presented here, one could perfectly well test General Relativity if some weaker coordination principle were introduced, one that left gravity and inertia distinct. One can compare to massive spin-2 gravity, which one would expect to have nearly the same empirical content as General Relativity (for sufficiently small graviton mass, making the natural assumption of a smooth limit as the graviton mass goes to 0) while differing radically from General Relativity on foundational issues (Freund 
et al. 1969). Thus it is clear that the empirical content of General Relativity resides in the partial differential equations of the theory, not an additional principle about gravity and inertia. At best the principle of equivalence (identifying gravity and inertia) might be a feature of the field equations of General Relativity, but it certainly does not needed to be added to the field equations.

A prima facie plausible philosophy of geometry for bimetric massive variants of (i.e., rivals to) General Relativity was outlined clearly by Freund, Maheshwari and Schonberg in the late 1960s in connection with their massive spin-2 gravitational theory (Freund et al. 1969). The job of coordination gets done, but not by Friedman's principle of equivalence, which is clearly false for massive spin-2 gravity. Rather it is done by the field equations, gravitational and material, of the theory. Such a theory, while strikingly different from Einstein's theory ontologically, approximates Einstein's theory arbitrarily well. That is precisely analogous to what happens in de Broglie-Proca massive electromagnetism. ${ }^{3}$ An illuminating but somewhat lengthy excerpt from that paper is included as an "Appendix". Here is a small portion:

\section{a) Breakdown of Geometrical Interpretation}

The theory, not being generally covariant, cannot be interpreted geometrically. This means first of all that the quadratic form,

$$
d \sigma^{2}=g_{\mu \nu} d x^{\mu} d x^{v},
$$

has nothing to do with the line element of the world geometry, which remains

$$
d s^{2}=\eta_{\mu v} d x^{\mu} d x^{v}
$$

...The geometrical interpretation is one of the crucial steps in applications of Einstein's theory. What do we offer as a replacement? The field equations... and the equations of motion for matter...fully determine the answer to any question one can ask....

\section{b) Local Problems}

If our theory is different from Einstein's, does this mean that it conflicts with the classical tests of the latter? No. All classical tests are local, i.e., they involve only small regions of space and time. Locally our theory differs from that of Einstein only by terms of the order (radius of system/Hubble radius),...(Freund et al. 1969)

Thus the principle of equivalence is not necessary for empirical content even in Einstein's theory (at least if the principle of equivalence is something over and above Einstein's field equations and their coordination to gravity and heavy matter). Today's philosophical reader will sense some affinity with Brown's space-time

\footnotetext{
3 Actually various devils in the details arise for massive gravity, but one is hardly entitled to appeal to those until and unless one takes massive gravity seriously enough to see the point, and then stares at it longer and harder to find devils in the details. It is not as if those who didn't entertain massive gravity somehow "knew all along" that it didn't work.
} 
philosophy (Brown 2005), especially because both attend to theories with more than one metric (Pitts 2016c).

As it turns out, if one thinks carefully and consistently about causality in massive gravity, matters get complicated (Pitts and Schieve 2007). In fact many facets of massive spin-2 gravity get subtle on close enough inspection, problems that do not arise in electromagnetism. But that is hardly a vindication of ignoring the theory and being (maybe) right for the wrong reason. Sometimes in cartoons one can be systematically lucky, as when unwittingly Bugs Bunny was chased by a hungry vampire in the latter's castle and happened to utter magic words at just the right times to avoid being bitten (Dunn 1963). But space-time philosophy is not a topic in which one cannot rationally plan to be lucky. One therefore needs to attend to alternative possibilities (unless one is Hegelian perhaps ${ }^{4}$ ).

\section{4 'Massive' Newtonian Gravity is Strictly Galilean}

There is an interesting irony for Kant's views on Newton's physics. Kant argues, as described by Michael Friedman, that we

need to presuppose the immediacy and universality of gravitational attraction in order to develop a rigorous method for comparing the masses of the primary bodies in the solar system. [footnote suppressed] We need such a method, in turn, in order rigorously to determine the center of mass of the solar system. (Friedman 1992, p. 157)

That is important because

...Kant does not have the concept of inertial frame and instead views the Newtonian laws of motion (together with other fundamental principles Kant takes to be a priori) as defining a convergent sequence of ever better approximations to a single privileged frame of reference (a counterpart of absolute space) at rest at the center of gravity of all matter. (Friedman 2001, p. 37)

Kant also appears to say [though on balance Friedman thinks otherwise (Friedman 1992 , pp. 166, 167)] that the $\frac{1}{r^{2}}$ law is a priori due to geometry.

Kant's view is, in light of twentieth century particle physics, almost backwards. A $\frac{1}{r^{2}}$ force comes from Laplace's equation in spherical symmetry in three spatial dimensions. But since Neumann's work it has become clear that there is a more general way to have a 3-dimensional equation akin to Laplace's, but with a new parameter-what one would now call a graviton mass. Laplace's equation is only appropriate for massless gravitons. If space is three-dimensional but the graviton has a small mass $m$, then gravity has instead a $\frac{e^{-m r}}{r}$ potential instead of $\frac{1}{r}$. The force is again given by the derivative of the potential. Thus the geometrical argument from the 3-dimensionality of space and solving a Laplace-like linear differential equation

\footnotetext{
${ }^{4}$ On Hegel, Lakatos and Feyerabend, see Motterlini (2002).
} 
excludes many possible force laws (including some of Seeliger's), but does not count against $\frac{e^{-m r}}{r}$. Hence a $\frac{1}{r}$ potential cannot be known a priori.

Things get a bit worse for Kant's views of what Newtonian gravity allows one to know. The symmetry group of Newtonian gravity is larger than the Galilean group and contains accelerations (Misner et al. 1973, p. 294; Smith 2008; Newton et al. 1999, p. 424). This isn't really news, given that Newton said as much (although Kant apparently struggled even with the Galilean relativity symmetry, to say nothing of the less famous symmetries larger than the Galilean group). Newton wrote:

Corollary 6. If bodies are moving in any way whatsoever with respect to one another and are urged by equal accelerative forces along parallel lines, they will all continue to move with respect to one another in the same way as they would if they were not acted on by those forces. (Smith 2008) (Newton et al. 1999, p. 424)

Hence one cannot tell using observations of the solar system whether the whole system is accelerating or not, much less whether it is at rest, pace Kant.

But massive graviton theories bring Kant some more good news: one can get more of what Kant wanted from Newton's theory if one uses Seeliger-NeumannEinstein "massive Newtonian gravity" [if the reader will permit the anachronism, which comes naturally to particle physicists (Boulware and Deser 1972)]. The graviton mass term, which is algebraic in the gravitational potential, destroys the symmetries beyond the Galilean group. Kant's lacking the concept of an inertial frame of reference and believing in a preferred frame in which the center of mass is at rest (Friedman 2001, p. 37) leave him destined for disappointment by any theory with a boost symmetry, whether Galilean or relativistic. But by having only the Galilean symmetry group, massive Newtonian gravity comes much closer to achieving Kant's goals than does Newton's theory. That Newton's theory isn't a necessary truth turns out to be perhaps a good thing for Kant.

\section{Conclusion}

One cannot rightly understand the actual philosophical significance of General Relativity, including the true rational force of its destructive impact on neoKantianism and the rationality of the views of the leader of the Vienna Circle, without attention to particle physics. If anything really made a neo-Kantian philosophy of geometry impossible (to a scholar who transcended the usual disciplinary boundaries but required no superhuman intelligence), it happened in the 1970s (van Dam and Veltman 1970, 1972; Boulware and Deser 1972), when massive spin-2 gravity died (or at any rate seemed to die). Finally one had to accept the conceptual innovations of General Relativity, half a century after Schlick had claimed so on much weaker grounds. This overthrow of Kant was entirely unheralded at the time. Few philosophers still cared by the early 1970s about a Kantian philosophy of geometry. Philosophers sought no guidance from particle 
physicists about space-time [despite its potential value by then (Freund et al. 1969)]. Particle physicists paid little attention to philosophy (Mermin 2004). But if the philosophy of geometry is not to be held captive by historical accidents, then the cause of death for a Kantian philosophy of geometry-in rationally reconstructed history!-involved the van Dam-Veltman-Zakharov discontinuity of massive pure spin-2 gravity in the limit of 0 graviton mass and the threat of instability [but see (Maheshwari 1972; Pitts 2016f) on the latter point]. This philosophical death is also apparently reversible, and perhaps now reversed (Deffayet et al. 2002; de Rham et al. 2011; Hassan and Rosen 2012; Hinterbichler 2012; de Rham 2014). Massive spin-2 gravity might live, at least for now. (So it has seemed to a fair number of working physicists within our own decade.) So might synthetic a priori knowledge live, if one wants it to. I do not write to defend it, but to show that physics has left the matter open until more recently than is generally believed, and that if and when physics forecloses the option, the grounds will be different from Schlick's. Of course the attention focussed on massive spin-2 gravity might wind up hastening its demise (Deser and Waldron 2013); if fatal objections are there to be found, they will be found faster now that people are looking. Maybe Kant's synthetic a priori knowledge is finally being scientifically refuted definitively a century after General Relativity appeared? Perhaps a good argument will vindicate Schlick's claims at last.

Particle physics has also proven useful recently in the historiography of General Relativity, shedding light on Einstein's invocation of energy conservation and on what was really wrong with his 1913 Entwurf theory (Pitts 2016b).

Acknowledgements I thank Marco Giovanelli, Dennis Lehmkuhl, and Jeremy Butterfield for assistance and discussion, Karl-Heinz Schlote for helping me to find some of Neumann's works, Don Howard for much useful instruction, and the anonymous referees for helpful comments. This work was supported by John Templeton Foundation grant \#38761.

Open Access This article is distributed under the terms of the Creative Commons Attribution 4.0 International License (http://creativecommons.org/licenses/by/4.0/), which permits unrestricted use, distribution, and reproduction in any medium, provided you give appropriate credit to the original author(s) and the source, provide a link to the Creative Commons license, and indicate if changes were made.

\section{Appendix: Philosophy of Geometry from Massive Spin 2 Gravity}

Freund, Maheshwari and Schonberg comment on their theory as follows:

It is the [non-generally covariant or bimetric part of the graviton mass] term that contains all the novel features of our theory. Without it the Lagrangian would lead to generally covariant field equations and as such would describe a massless field [i.e., General Relativity]. It is only the presence of this one term that breaks general covariance. The departures from Einstein's theory can now easily be identified. 


\section{a) Breakdown of Geometrical Interpretation}

The theory, not being generally covariant, cannot be interpreted geometrically. This means first of all that the quadratic form,

$$
d \sigma^{2}=g_{\mu \nu} d x^{\mu} d x^{\nu},
$$

has nothing to do with the line element of the world geometry, which remains

$$
d s^{2}=\eta_{\mu v} d x^{\mu} d x^{v} .
$$

Similarly, the equations of motion of matter...still look formally as if they were geodesic equations. As a matter of fact, they are not. Indeed, the $\Gamma_{\mu \nu}^{\sigma}$ are given by the usual expressions, but $g_{\mu \nu}$ and... [its inverse] are determined from the not-generally-covariant [field] equations..., so that the $\Gamma_{\mu \nu}^{\sigma}$ are not genuine Christoffel symbols. The geometrical interpretation is one of the crucial steps in applications of Einstein's theory. What do we offer as a replacement? The field equations... and the equations of motion for matter...fully determine the answer to any question one can ask. For that matter, this is true for Einstein's theory as well. There, however, geometrical considerations may be used as a luxurious shortcut toward the answers to many problems.

\section{b) Local Problems}

If our theory is different from Einstein's, does this mean that it conflicts with the classical tests of the latter? No. All classical tests are local, i.e., they involve only small regions of space and time. Locally our theory differs from that of Einstein only by terms of the order (radius of system/Hubble radius), so that the corrections are indeed negligible and the local tests cannot distinguish between the two theories. Moreover, locally one can reinstate an approximate geometrical interpretation. One may wonder whether there is any sense to an approximate gauge invariance. Fortunately, there is a test case available in nature: chiral gauge invariance. Even though the breaking of the gauge invariance occurs through a mass as large as that of the $\pi$-meson, the lowenergy theorems that follow from the chiral-gauge group are still valid to a very good degree of accuracy. It is thus totally justified to expect the lowenergy theorems of Einstein's theory to hold to a much better accuracy as $\Lambda / m_{\pi}^{2}=O\left(10^{-80}\right)$. Thus at a local level our theory is indistinguishable by usual experiments from that of Einstein. The real difference appears for systems of the size of $\Lambda^{-\frac{1}{2}}$, that is, for cosmological problems. ...Here let us only emphasize once more that ours is a theory in flat space. The pseudoEuclidean metric can be observed only in cosmological experiments. Local experiments could detect it only if performed accurately enough to be sensitive to terms of the order (size of system/Hubble radius). (Freund et al. 1969)

As noted above, there arose devils in the details in the early 1970s, which might or might not have been exorcised recently. Exactly this relationship holds, however, in 
the simpler scalar case between massless spin 0 (Nordström's 1914 theory) and massive scalar gravity (Boulware and Deser 1972; Pitts 2011a, 2016d), as Seeliger expected in the already 19 th century.

\section{References}

Belinfante, F. J. (1949). The interaction representation of the Proca field. Physical Review, 76, 66-80. Boulware, D. G., \& Deser, S. (1972). Can gravitation have a finite range? Physical Review D, 6, 3368-3382.

Brink, L. (2006). A non-geometric approach to 11-dimensional supergravity. In J. T. Liu, M. J. Duff, K. S. Stelle, \& R. P. Woodard (Eds.), Deserfest: A celebration of the life and works of Stanley Deser (pp. 40-54). Hackensack, NJ: World Scientific.

Brown, H. R. (2005). Physical relativity: Space-time structure from a dynamical perspective. New York: Oxford University Press.

Brush, S. G. (1989). Prediction and theory evaluation: The case of light bending. Science, 246, 1124-1129.

Carnap, R. (1963). Adolf Grünbaum on the philosophy of space and time. In P. A. Schilpp (Ed.), The philosophy of Rudolf Carnap (pp. 952-958). La Salle, IL: Open Court.

Coffa, J. A. (1991). The semantic tradition from Kant to Carnap: To the Vienna station. Cambridge: Cambridge University Press. (edited by Linda Wessels).

Combridge, J. T. (1965). Bibliography of relativity and gravitation theory 1921-1937. London: King's College London.

de Broglie, L. (1922). Rayonnement noir et quanta de lumière. Journal de Physique et la Radium, 3, 422-428.

de Broglie, L. (1923). Ondes et quanta. Comptes Rendus Hebdomadaires des Séances de l'Académie des Sciences, 177, 507-510.

de Broglie, L. (1924). A tentative theory of light quanta. Philosophical Magazine and Journal of Science, 47, 446-458.

de Broglie, L. (1943). Théorie Général des Particules a Spin (Method de Fusion). Paris: Gauthier-Villars.

de Broglie, L. (1954). Théorie Général des Particules a Spin (Method de Fusion) (2nd ed.). Paris: Gauthier-Villars.

de Rham, C. (2014). Massive gravity. Living Reviews in Relativity, 17(7). arXiv:1401.4173v2 [hep-th].

de Rham, C., Gabadadze, G., \& Tolley, A. J. (2011). Resummation of massive gravity. Physical Review Letters, 106, 231101. arXiv:1011.1232v2 [hep-th].

Deffayet, C., Dvali, G., Gabadadze, G., \& Vainshtein, A. I. (2002). Nonperturbative continuity in graviton mass versus perturbative discontinuity. Physical Review D, 65, 044026. hep-th/0106001v2.

Deser, S. (1970). Self-interaction and gauge invariance. General Relativity and Gravitation, 1, 9-18. arXiv:gr-qc/0411023v2.

Deser, S. (1971). Mixing and mass in spin 2 systems. In A. Salam, M. Dal Cin, G. J. Iverson, \& A. Perlmutter (Eds.), Nonpolynomial lagrangians, renormalisation and gravity, volume 1 of Center for Theoretical Studies, University of Miami, tracts in mathematics and natural sciences (pp. 106-111). New York: Gordon and Breach Science.

Deser, S., \& Waldron, A. (2013). Acausality of massive gravity. Physical Review Letters, 110, 111101. arXiv: 1212.5835 .

Duhem, P. (1954). The aim and structure of physical theory. Princeton: Princeton University. Translated from the second French edition, 1914; original 1906.

Dunn, J. (1963). Transylvania 6-5000. http://www.bcdb.com/cartoon_video/5243-Transylvania_6-5000. html.

Earman, J. (1992). Bayes or bust? A critical examination of Bayesian confirmation theory. Cambridge: Massachusetts Institute of Technology Press.

Earman, J. (2001). Lambda: The constant that refuses to die. Archive for History of Exact Sciences, 55, $189-220$.

Eddington, A. (1920). Space, time and gravitation: An outline of the general relativity theory. Cambridge: Cambridge University Press. 
Eddington, A. S. (1913). Jules Henri Poincare [Obituary]. Monthly Notices of the Royal Astronomical Society, 73(4), 223-228.

Eddington, A. S. (1924). A comparison of Whitehead's and Einstein's formulae. Nature, 113, 192.

Einstein, A. (1923). Cosmological considerations on the general theory of relativity. In H. A. Lorentz, A. Einstein, H. Minkowski, H. Weyl, A. Sommerfeld, W. Perrett, \& G. B. Jeffery (Eds.), The principle of relativity (pp. 175-188). Methuen, London. Dover reprint, New York, 1952. Translated from Kosmologische Betrachtungen zur allgemeinen Relativitätstheorie. Sitzungsberichte der Königlich Preussichen Akademie der Wissenschaften zu Berlin (1917) pp. 142-152.

Einstein, A., \& Fokker, A. D. (1914). Die Nordströmsche Gravitationstheorie vom Standpunkt des absoluten Differentialkalküls. Annalen der Physik, 44, 321-328. English translation in Anna Beck and Don Howard, The Collected Papers of Albert Einstein, Volume 4, The Swiss Years: Writings, 1912-1914. English Translation, The Hebrew University of Jerusalem and Princeton University Press, Princeton, 1996, 293-299.

Feynman, R. P., Morinigo, F. B., Wagner, W. G., Hatfield, B., Preskill, J., \& Thorne, K. S. (1995). Feynman lectures on gravitation. Reading, MA: Addison-Wesley. Original by California Institute of Technology, 1963.

Fierz, M., \& Pauli, W. (1939). On relativistic wave equations for particles of arbitrary spin in an electromagnetic field. Proceedings of the Royal Society (London) A, 173, 211-232.

Freund, P. G. O., Maheshwari, A., \& Schonberg, E. (1969). Finite-range gravitation. Astrophysical Journal, 157, 857-867.

Friedman, M. (1992). Kant and the exact sciences. Cambridge: Harvard University Press.

Friedman, M. (2001). Dynamics of reason: The 1999 Kant Lectures at Stanford University. Stanford: CSLI Publications.

Friedman, M. (2002). Kant, Kuhn, and the rationality of science. Philosophy of Science, 69, 171-190.

Gupta, S. N. (1954). Gravitation and electromagnetism. Physical Review, 96, 1683-1685.

Hacking, I. (2000). How inevitable are the results of successful science? Philosophy of Science, 67, S58S71.

Harvey, A., \& Schucking, E. (2000). Einstein's mistake and the cosmological constant. American Journal of Physics, 68(8), 723-727.

Hassan, S. F., \& Rosen, R. A. (2011). On non-linear actions for massive gravity. Journal of High Energy Physics, 1107(009). arXiv:1103.6055v3 [hep-th].

Hassan, S. F., \& Rosen, R. A. (2012). Confirmation of the secondary constraint and absence of ghost in massive gravity and bimetric gravity. Journal of High Energy Physics, 1204(123), arXiv:1111.2070 [hep-th].

Heckmann, O. (1942). Theorien der Kosmologie. Berlin: Springer, revised edition. Reprinted 1968.

Hentschel, K. (1987). Einstein, Neokantianismus und Theorienholismus. Kant-Studien, 78, 459-470.

Hentschel, K. (1990). Interpretationen und Fehlinterpretationen der speziellen und der allgemeinen Relativätstheorie durch Zeitgenossen Albert Emsteins. Basel: Birkhäuser Verlag.

Hinterbichler, K. (2012). Theoretical aspects of massive gravity. Reviews of Modern Physics, 84, 671-710. arXiv:1105.3735v2 [hep-th].

Howard, D. (1984). Realism and conventionalism in Einstein's philosophy of science: The EinsteinSchlick correspondence. Philosophia Naturalis, 21, 618-629.

Howard, D. (1994). Einstein, Kant, and the Origins of Logical Empiricism. In W. Salmon \& G. Wolters (Eds.), Logic, language, and the structure of scientific theories: Proceedings of the CarnapReichenbach Centennial, University of Konstanz, 21-24 May 1991 (pp. 45-105). Pittsburgh/ Konstanz: University of Pittsburgh Press/Universitätsverlag Konstanz.

Howard, D. (2010). Let me briefly indicate why I do not find this standpoint natural. Einstein, General Relativity, and the contingent a priori. In M. Dickson \& M. Domski (Eds.), Discourse on a new method: Reinvigorating the marriage of history and philosophy of science (pp. 333-355). Chicago: Open Court.

Jammer, M. (1993). Concepts of space (3rd ed.). New York: Dover.

Kerszberg, P. (1989). The invented universe: The Einstein-De Sitter controversy (1916-17) and the rise of relativistic cosmology. Oxford: Clarendon Press.

Khalifa, K. (2010). Default privilege and bad lots: Underconsideration and explanatory inference. International Studies in the Philosophy of Science, 24, 91-105.

Kragh, H. (1984). Equation with the many fathers: The Klein-Gordon equation in 1926. American Journal of Physics, 52, 1024-1033. 
Kragh, H. (2004). Matter and spirit in the universe: Scientific and religious preludes to modern cosmology. London: Imperial College.

Kraichnan, R. H. (1955). Special-relativistic derivation of generally covariant gravitation theory. Physical Review, 98, 1118-1122.

Kuhn, T. S. (1971). Notes on lakatos. In R. C. Buck \& R. S. Cohen (Eds.), PSA: Proceedings of the biennial meeting of the philosophy of science association, 1970, Boston studies in the philosophy of science (pp. 137-146). Dordrecht: D. Reidel.

Lakatos, I. (1970). Falsification and the methodology of scientific research programmes. In I. Lakatos \& A. Musgrave (Eds.), Criticism and the growth of knowledge (pp. 91-196). London: Cambridge University Press.

Lakatos, I. (1971). History of science and its rational reconstruction. In R. C. Buck \& R. S. Cohen (Eds.), PSA: Proceedings of the biennial meeting of the philosophy of science association, 1970, Boston studies in the philosophy of science (pp. 91-136). Dordrecht: D. Reidel.

Levi-Civita, T. (1926). The absolute differential calculus: Calculus of tensors. London: Blackie and Son. Translated by Marjorie Long.

Lotze, H. (1879). Metaphysik. Drei Bücher der Ontologie, Kosmologie, und Psychologie. S. Hirzel, Leipzig. Bibliothèque nationale de France, gallica.bnf.fr.

Maheshwari, A. (1972). Spin-2 field theories and the tensor-field identity. Il Nuovo Cimento, 8A, 319-330.

McCrea, W. H. (1971). The cosmical constant. Quarterly Journal of the Royal Astronomical Society, 12, 140-153.

Mermin, N. D. (2004). Could Feynman have said this? Physics Today, 57(5), 10, 11.

Misner, C., Thorne, K., \& Wheeler, J. A. (1973). Gravitation. New York: Freeman.

Mittelstaedt, P. (1970). Lorentzinvariante Gravitationstheorie. Westdeutscher, Cologne. From meeting on 3 December 1969.

Mittelstaedt, P. (1976). Philosophical problems of modern physics. Dordrecht: Reidel. Translated from the revised fourth edition by Dr. W. Riemer, 1972.

Motterlini, M. (2002). Reconstructing Lakatos: A reassessment of Lakatos epistemological project in the light of the Lakatos Archive. Studies in History and Philosophy of Science, 33, 487-509.

Neumann, C. (1896). Allgemeine Untersuchungen über das Newton'sche Princip der Fernwirkungen mit besonderer Rücksicht auf die Elektrischen Wirkungen. Leipzig: B. G. Teubner.

Neumann, C. (1903). Über eine gewisse Gattung von Kugelflächen-Integralen. Berichte über die Verhandlungen der Königlich Säschsichen Gesellschaft der Wissenschaften zu Leipzig, Mathematisch-Physische Klasse, 55, 264-286.

Neumann, C. G. (1886). Ueber gewisse particulare Integrale der Differentialgleichung $\Delta F=F$, insbesondere über die Entwickelung dieser particularen Integrale nach Kugelfunctionen. Berichte über die Verhandlungen der Königlich Sächsischen Gesellschaft der Wissenschaften zu Leipzig. Mathematisch-Physische Classe, 38, 75-82.

Newton, I., Cohen, I. B., Whitman, A., \& Budenz, J. (1999). The principia: Mathematical principles of natural philosophy. Berkeley: University of California.

North, J. (1994). The Norton history of astronomy and cosmology. New York: W. W. Norton \& Company.

North, J. D. (1965). The measure of the universe: A history of modern cosmology. Oxford University, 1990 Dover reprint, New York.

Norton, J. D. (1999). The cosmological woes of Newtonian gravitation theory. In H. Goenner, J. Renn, J. Ritter, \& T. Sauer (Eds.), The expanding worlds of general relativity, Einstein studies (Vol. 7, pp. 271-323). Boston: Birkhäuser.

Ogievetsky, V. I., \& Polubarinov, I. V. (1965). Interacting field of spin 2 and the Einstein equations. Annals of Physics, 35, 167-208.

Ohanian, H. C. (2008). Einstein's mistakes: The human failings of genius. New York: W. W. Norton \& Company.

Okruhlik, K. (2004). Logical empiricism, feminism, and Neurath's auxiliary motive. Hypatia, 19, 48-72.

Pais, A. (1982). 'Subtle is the Lord...': The science and the life of Albert Einstein. Oxford: Oxford University Press.

Pauli, W., \& Fierz, M. (1939). Über relativistische Feldgleichungen von Teilchen mit beliebigem Spin im elektromagnetischen Feld. Helvetica Physica Acta, 12, 297-300.

Petiau, G. (1941a). Sur la représentation unitaire de l'électromagnétisme et de la gravitation en mécanique ondulatoire. Comptes rendus hebdomadaires des séances de l'Académie des sciences, 212, 1126-1128. 
Petiau, G. (1941b). Sur la théorie du corpuscule de spin 2. Comptes rendus hebdomadaires des séances de l'Académie des sciences, 212, 292-295.

Petiau, G. (1945). Sur les interactions entre particules matérielles s'exerçant par l'intermédiaire de la particule de spin $2 \frac{h}{2 \pi}$. Journal de Physique et le Radium, 6, 115-120.

Pitts, J. B. (2011a). Massive Nordström scalar (density) gravities from universal coupling. General Relativity and Gravitation, 43, 871-895. arXiv:1010.0227v1 [gr-qc].

Pitts, J. B. (2011b). Permanent underdetermination from approximate empirical equivalence in field theory: Massless and massive scalar gravity, neutrino, electromagnetic, Yang-Mills and gravitational theories. The British Journal for the Philosophy of Science, 62, 259-299.

Pitts, J. B. (2013). Irrelevant conjunction and the ratio measure or historical skepticism. Synthese, 190, 2117-2139.

Pitts, J. B. (2016a). Einstein's equations for spin 2 mass 0 from Noether's converse Hilbertian assertion. Studies in History and Philosophy of Modern Physics, 56, 60-69. arXiv:1611.02673 [physics.hist$\mathrm{ph}$.

Pitts, J. B. (2016b). Einstein's physical strategy, energy conservation, symmetries, and stability: "But Grossmann \& I believed that the conservation laws were not satisfied". Studies in History and Philosophy of Modern Physics, 54, 52-72. arXiv:org/1604.03038 [physics.hist-ph].

Pitts, J. B. (2016c). Space-time constructivism vs. modal provincialism: Or, How special relativistic theories needn't show Minkowski chronogeometry. forthcoming in Studies in History and Philosophy of Modern Physics as invited reviewed contribution to special issue on Harvey Brown's Physical Relativity 10 years later, edited by Simon Saunders.

Pitts, J. B. (2016d). Space-time philosophy reconstructed via massive Nordström scalar gravities? Laws vs. geometry, conventionality, and underdetermination. Studies in History and Philosophy of Modern Physics, 53, 73-92. arXiv:1509.03303 [physics.hist-ph].

Pitts, J. B. (2016e). Underconsideration in space-time and particle physics. http://philsci-archive.pitt.edu/ $12831 /$.

Pitts, J. B. (2016f). Universally coupled massive gravity, III: dRGT-Maheshwari pure spin-2, OgievetskyPolubarinov and arbitrary mass terms. Annals of Physics, 365, 73-90. arXiv:1505.03492 [gr-qc].

Pitts, J. B., \& Schieve, W. C. (2001). Slightly bimetric gravitation. General Relativity and Gravitation, 33, 1319-1350. arXiv:gr-qc/0101058v3.

Pitts, J. B., \& Schieve, W. C. (2007). Universally coupled massive gravity. Theoretical and Mathematical Physics, 151, 700-717. arXiv:gr-qc/0503051v3.

Pockels, F. (1891). Über die Partielle Differentialgleichung $\Delta u+k^{2} u=0$ und deren Auftreten in der mathematischen Physik. Leipzig: B. G. Teubner.

Poincaré, H. (1902). La Science et l'hypothèse. Ernest Flammarion, Paris. translation Science and Hypothesis by W. J. G., Walter Scott Publishing, 1905; reprint Dover, New York, 1952.

Poincaré, H. (1913). Science and Hypothesis. In The Foundations of Science. The Science Press, Lancaster, Pennsylvania. Translated by George Bruce Halsted, reprinted 1946; French original 1902.

Reichenbach, H. (1928). Philosophie der Raum-Zeit-Lehre. Braunschweig: Vieweg.

Reichenbach, H. (1929a). Die neue Theorie Einsteins über die Verschmelzung von Gravitation und Elektrizität. Zeitschrift für angewandte Chemie, 42, 121-123.

Reichenbach, H. (1929b). Zur Einordnung des neuen Einsteinschen Ansatzes über Gravitation und Elektrizität. Zeitschrift für Physik, 53, 683-689.

Reichenbach, H. (1958). The Philosophy of Space and Time. Dover, New York. Partial translation by Maria Reichenbach and John Freund of Philosophie der Raum-Zeit-Lehre, Vieweg, Braunschweig (1928).

Reichenbach, H. (1978). The present state of the discussion of relativity. In Reichenbach, M. \& Cohen, R. S. (Eds.), Selected writings, 1909-1953 (Vol. 2, pp. 3-47). Dordrecht: D. Reidel. Translated by Maria Reichenbach from 1922 original.

Reichenbach, H. (n.d.). Weyl's extension of the Riemannian concept of space and the geometrical interpretation of electricity - appendix. Box 41, Folder 21. http://digital.library.pitt.edu/u/ ulsmanuscripts/pdf/31735062233568.

Reichenbach, H., Gimbel, S., \& Walz, A. (2006). Defending Einstein: Hans Reichenbach's writings on space, time, and motion. Cambridge: Cambridge University Press.

Roush, S. (2005). Tracking truth: Knowledge, evidence, and science. Oxford: Clarendon Press.

Rovelli, C. (2002). Notes for a brief history of quantum gravity. In R. T. Jantzen, R. Ruffini, \& V. G. Gurzadyan (Eds.), Proceedings of the Ninth Marcel Grossmann Meeting (held at the University of 
Rome “La Sapienza,” 2-8 July 2000) (pp. 742-768). River Edge, NJ: World Scientific. arXiv:gr-qc/ 0006061.

Russell, B. (1927). The analysis of matter. Kegan Paul, Trench, Trubner \& Co., London; Harcourt, Brace and Company, New York.

Ryckman, T. (2005). The reign of relativity: Philosophy in physics 1915-1925. Oxford: Oxford University Press.

Salmon, W. C. (1977). The philosophy of Hans Reichenbach. Hans Reichenbach: Logical empiricist. Dordrecht: D. Reidel.

Schild, A. (1956). On gravitational theories of Whitehead's type. Proceedings of the Royal Society of London A: Mathematical and Physical Sciences, 235, 202-209.

Schlick, M. (1915). Die philosophische Bedeutung des Relativitätsprinzips. Zeitschrift für Philosophie und philosophische Kritik, 159, 129-175. Translated by Peter Heath as "The Philosophical Significance of the Principle of Relativity" in Henk L. Mulder and Barbara F. B. van de VeldeSchlick, editors, Moritz Schlick Philosophical Papers, Volume I (1909-1922), pp. 153-189. D. Reidel, Dordrecht (1979).

Schlick, M. (1920). Space and time in contemporary physics. Oxford University. Translated by Henry L. Brose; reprint Dover, New York, 1963.

Schlick, M. (1921). Kritische oder empiristische Deutung der neuen Physik? Kant-Studien, 26, 96-111. Translated by Peter Heath as "Critical or Empiricist Interpretation of Modern Physics?" in Henk L. Mulder and Barbara F. B. van de Velde-Schlick, editors, Moritz Schlick Philosophical Papers, Volume I (1909-1922), pp. 322-334. D. Reidel, Dordrecht (1979).

Schucking, E. L. (1991). The introduction of the cosmological constant. In A. Zichichi, V. de Sabbata, \& N. Sánchez (Eds.), Gravitation and modern cosmology: The cosmological constant problem (pp. 185-187), honor of Peter Gabriel Bergmann's 75th birthday Plenum: New York.

Shimony, A. (1970). Scientific inference. In R. G. Colodny (Ed.), The nature \& function of scientific theories (Vol. 4, pp. 79-172). Pittsburgh: University of Pittsburgh Series in the Philosophy of Science University of Pittsburgh Press.

Sklar, L. (1985). Do unborn hypotheses have rights? In Philosophy and spacetime physics (pp. 148-166). Berkeley: University of California.

Smith, G. (2008). Newton's Philosophiae Naturalis Principia Mathematica. In E. N. Zalta (Ed.), The stanford encyclopedia of philosophy. Winter edition. http://plato.stanford.edu/archives/win2008/ entries/newton-principia/.

Sober, E. (2008). Evidence and evolution: The logic behind the science. Cambridge: Cambridge University Press.

Soler, L. (2008). Revealing the analytical atructure and some intrinsic major difficulties of the contingentist/inevitabilist issue. Studies in History and Philosophy of Science, 39, 230-241.

Stanford, P. K. (2006). Exceeding our grasp: Science, history, and the problem of unconceived alternatives. New York: Oxford University.

Tonnelat, M.-A. (1941). Sur les ondes planes de la particule de spin 2 (graviton). Comptes rendus hebdomadaires des séances de l'Académie des sciences, 212, 263-266.

Tonnelat, M.-A. (1943). Théorie euclidienne de l'électromagnétisme et de la gravitation. Disquisitiones Mathematicae et Physicae, 3, 249-274.

Tonnelat, M.-A. (1944a). La particule de spin 2 et la loi de gravitation d'Einstein dans le cas de présence de matière. Comptes rendus hebdomadaires des séances de l'Académie des sciences, 218, 305-308.

Tonnelat, M.-A. (1944b). Les phénomènes de gravitation. Étude des interactions entre la matiére et la particule de spin 2. Annales de Physique, 19, 396-445.

Tonnelat, M.-A. (1944c). Sur l'interaction entre deux particules matérielles au moyen du corpuscule de spin maximum 2; loi de gravitation newtonienne. Comptes rendus hebdomadaires des séances de l'Académie des sciences, 218, 139-141.

Torretti, R. (1978a). Hugo Dingler's philosophy of geometry. Diálogos, 32, 85-128.

Torretti, R. (1978b). Philosophy of geometry from Riemann to Poincaré. Dordrecht: D. Reidel.

Trautman, A. (1965). Foundations and current problems of General Relativity. In S. Deser \& K. W. Ford (Eds.), Lectures on general relativity (pp. 1-248). Englewood Cliffs, NJ: Prentice Hall. Brandeis Summer Institute in Theoretical Physics.

Tyutin, I. V., \& Fradkin, E. S. (1972). Quantization of massive gravitation. Soviet Journal of Nuclear Physics, 15, 331-334.

Vainshtein, A. I. (1972). To the problem of nonvanishing gravitation mass. Physics Letters B, 39, 393-394. 
van Dam, H., \& Veltman, M. (1970). Massive and mass-less Yang-Mills and gravitational fields. Nuclear Physics B, 22, 397-411.

van Dam, H., \& Veltman, M. (1972). On the mass of the graviton. General Relativity and Gravitation, 3, $215-220$.

van Fraassen, B. (1989). Laws and symmetry. Oxford: Clarendon Press.

von Laue, M. (1917). Die Nordströmsche Gravitationstheorie. Journal der Radioaktivität und Electronik, 14, 263-313.

von Seeliger, H. (1895). Ueber das Newton'sche Gravitationgesetz. Astronomische Nachrichten, 137, 129-136. NASA ADS.

von Seeliger, H. (1896). Ueber das Newton'sche Gravitationgesetz. Sitzungsberichte der mathematischphysikalischen Classe der k. b. Akademie der Wissenschaften zu München, 26, 373-400.

Walter, S. A. (2010). Moritz Schlick's reading of Poincaré's theory of relativity. In F. O. Engler \& M. Iven (Eds.), Moritz Schlick: Ursprünge und Entwicklungen seines Denkens (Vol. 5, pp. 191-203). Berlin: Schlickiana Parerga.

Weinberg, S. (1964a). Derivation of gauge invariance and the equivalence principle from Lorentz invariance of the S-matrix. Physics Letters, 9, 357-359.

Weinberg, S. (1964b). Photons and gravitons in $S$-matrix theory: Derivation of charge conservation and equality of gravitational and inertial mass. Physical Review, 135, B1049-B1056.

Whitehead, A. N. (1922). The principle of relativity: With applications to physical science. Cambridge: Cambridge University Press.

Wigner, E. (1939). On unitary representations of the inhomogeneous Lorentz group. Annals of Mathematics, 40, 149-204.

Wray, K. B. (2008). The argument from underconsideration as grounds for anti-realism: A defence. International Studies in the Philosophy of Science, 22, 317-326.

Yukawa, H. (1935). On the interaction of elementary particles. I. Proceedings of the MathematicoPhysical Society of Japan, 17, 48-57. 